\title{
Combining High Angular Resolution Interferometry and Spectroscopy in Studies of Stars and Stellar Systems
}

\author{
John Davis \\ Chatterton Astronomy Department, School of Physics, University of \\ Sydney, NSW 2006, Australia
}

\begin{abstract}
A number of long baseline optical/infrared interferometers have commenced their scientific programs or are under development. These instruments will provide accurate measurements of the angular sizes of single stars and the angular separations of binary systems at resolutions impossible with conventional telescopes. The combination of interferometric data with the results of high-resolution spectroscopy will, for many classes of objects, provide a powerful method for studying them that neither technique can do alone. Examples include the combination of interferometric and spectroscopic data for spectroscopic binaries and, in particular, for double-lined binaries, and single-lined binaries of known parallax, to determine fundamental stellar quantities. Another example concerns the study of Cepheid variables, where the combination of the data can provide an independent calibration of the zero point of the luminosity scale. The requirements and potential of these combined interferometric-spectroscopic studies are discussed.
\end{abstract}

\section{Introduction}

The combination of interferometric and spectroscopic data is a powerful tool for the determination of fundamental stellar properties and for a range of studies in stellar astrophysics. Although it is nearly 80 years since Michelson and Pease (1921) measured the first stellar angular diameter, and nearly 30 years since Herbison-Evans et al. (1971) demonstrated the power of combining interferometric and spectroscopic data for the binary star $\alpha$ Vir, it is only now that we are in a position to really exploit the potential of combining these data. There are now several high angular resolution interferometers and interferometric arrays that have commenced or are commencing their scientific programs, and there are others currently being developed. It is therefore an appropriate time to review the potential of this combination of data.

\section{Programs for Interferometry}

Potential programs for high angular resolution optical and infrared interferometry have been reviewed by Davis (1979) and McAlister $(1979,1985)$. Table 1 contains a list of several of the potential stellar programs. 
Table 1. Programs for Interferometry

\begin{tabular}{|c|c|}
\hline Program & Aims \\
\hline Single stars & $\begin{array}{l}\text { Measurement of angular diameters leading to emergent fluxes, } \\
\text { effective temperatures and, if the parallaxes are known, } \\
\text { radii and luminosities }\end{array}$ \\
\hline Binary stars & $\begin{array}{l}\text { Measurements of angular diameters and separations of the } \\
\text { components of spectroscopic binaries leading to masses, radii } \\
\text { and luminosities simultaneously for individual stars, and } \\
\text { distances to the systems }\end{array}$ \\
\hline Pulsating stars & $\begin{array}{l}\text { Measurement of angular diameters and variations in angular } \\
\text { diameter leading to effective temperatures, radii, luminosities } \\
\text { and distances (e.g. for Cepheids and Miras) }\end{array}$ \\
\hline Extended envelopes and shells & $\begin{array}{l}\text { Measurement of sizes and shapes of emission regions relative } \\
\text { to the stellar diameter }\end{array}$ \\
\hline Stellar rotation & $\begin{array}{l}\text { Investigation of the effect of rotation on shape and brightness } \\
\text { distribution }\end{array}$ \\
\hline Limb darkening & $\begin{array}{l}\text { Determination of limb darkening via precise and multi-colour } \\
\text { observations }\end{array}$ \\
\hline Imaging & Stars and stellar systems \\
\hline Global (large angle) astrometry & $\begin{array}{l}\text { Maintenance of the Hipparcos frame and improved proper } \\
\text { motions }\end{array}$ \\
\hline Narrow angle astrometry & Binary systems and extrasolar planets \\
\hline
\end{tabular}

In addition to stellar programs there is naturally great interest in the possibility of using interferometry for galactic and extra-galactic studies. Examples of these are discussed in the proceedings of a workshop on "Science with the VLT Interferometer" (Paresce, 1997). However, the interferometers currently operating, or coming on line in the near future, are limited by their sensitivity to stellar programs. It follows that stellar programs are of more immediate interest and the present discussion is limited to them.

All the programs in Table 1 require complementary data obtained by photometry, spectrophotometry, astrometry or spectroscopy to fulfil their aims. The two programs for which high precision spectroscopy is of vital importance are those for binary and pulsating stars and these will be discussed in some detail.

\subsection{Spectroscopic Binary Stars}

The interferometric response to a binary star is shown in Figure 1. The quantity measured is generally the square of the interferometric fringe visibility, otherwise termed the correlation, as a function of the separation of the apertures (the baseline of the interferometer). The dashed envelope in Figure 1 is the response for a single star, or for the binary when the orientation is such that the angular separation projected onto the interferometer baseline is zero. The horizontal scale of the envelope is determined primarily by the angular size of the primary. The secondary will modify the shape of the envelope to an extent that depends on its relative brightness and angular diameter. The solid curve is a typical response whose characteristics are determined as follows. The depth of the modulation is determined by the relative brightness of the two components and the spatial frequency of the modulation is determined by the angular separation of the components projected onto the baseline of the interferometer. The projected angular separation varies with time, not only because of the orbital motion of the system, but also due to the variation of parallactic angle with hour angle. 


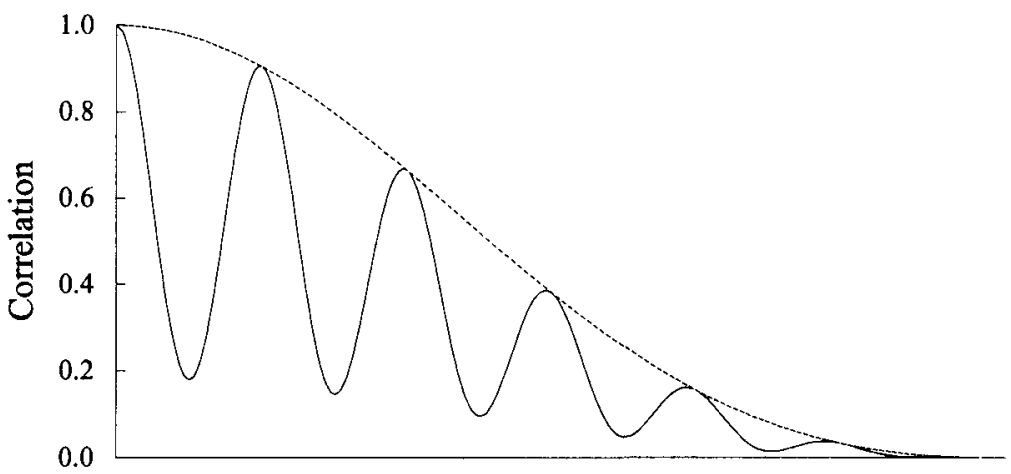

Baseline

Figure 1. The response of a two aperture interferometer to a binary star as a function of the separation of the apertures (the baseline of the interferometer). The features of this response are described in the text.

Extended observations at a fixed baseline will therefore exhibit a variation with time as the projected angular separation changes, and this allows the angular separation and position angle of the system, and the variation in these quantities as a consequence of orbital motion, to be determined. Examples of the observed variation of correlation with time at fixed baselines for binary systems can be found in the papers by Herbison-Evans et al. (1971), Pan et al. $(1990,1992)$ and Armstrong et al. (1992).

The majority of the orbital parameters of a spectroscopic binary system may be obtained either by interferometry or by spectroscopy. However, the one crucial parameter that spectroscopy cannot determine, namely the inclination of the orbit, can be obtained by interferometry just as it can for a visual binary. Interferometers with separated apertures have higher angular resolution than visual, speckle interferometry, and non-redundant masking interferometry observations. In many cases this will allow not only the orbit to be resolved but, by making observations at more than one baseline, it will also permit the determination of the angular size of at least the primary component and, in favourable cases, the angular size of the secondary component as well. Table 2 indicates the contributions of the various techniques to the determination of fundamental stellar properties. In the case of double-lined spectroscopic binaries (DLSBs), spectroscopy, interferometry, plus the flux distribution received at the Earth corrected for interstellar extinction, is all that is required to determine the fundamental properties given in Table 2. For single-lined spectroscopic binaries (SLSBs) the distance to the system is also needed. The advent of Hipparcos parallaxes (ESA 1997) will enable many single-lined systems to be included in a program of fundamental property determinations.

To date the only known published cases of combining interferometric and spectroscopic data for DLSBs, in which the angular diameters of one or both components have been measured, are for $\alpha$ Vir (Herbison-Evans et al. 1971) and a Aur (Hummel et al. 1994). These very different cases serve as illustrations 
Table 2. The determination of fundamental stellar properties from spectroscopic binary systems. Key: $m_{1,2}, R_{1,2}, T_{e 1,2}, L_{1,2}$ masses, radii, effective temperatures and luminosities of primary and secondary, $i$ inclination of orbit, $a$ semi-major axis, $\theta_{a}$ angular semi-major axis, $\theta_{1,2}$ angular diameters of primary and secondary, $f_{\lambda}$ flux/unit bandwidth received at earth (corrected for inter-stellar extinction), $\boldsymbol{d}$ distance. The parameters in parentheses for the secondary can only be determined for favourable systems.

\begin{tabular}{clll}
\hline $\begin{array}{c}\text { Type of } \\
\text { Binary }\end{array}$ & \multicolumn{1}{c}{ Technique } & \multicolumn{1}{c}{$\begin{array}{c}\text { Contributed } \\
\text { Data }\end{array}$} & \multicolumn{1}{c}{ Results } \\
\hline Double-lined & $\begin{array}{l}\text { Spectroscopy } \\
\text { Interferometry }\end{array}$ & $\begin{array}{l}m_{1} \sin ^{3} i, m_{2} \sin ^{3} i, a \sin i \\
\theta_{a}, i, \theta_{1}\left(\theta_{2}\right)\end{array}$ & $\begin{array}{l}m_{1}, m_{2}, a, d, R_{1}, T_{e 1}, L_{1} \\
\left(R_{2}, T_{e 2}, L_{2}\right)\end{array}$ \\
& Photometry & Integrated flux $\int_{0}^{\infty} f_{\lambda} d \lambda$ & \\
\hline \multirow{2}{*}{ Single-lined } & Spectroscopy & $\frac{\left(m_{2} \sin i\right)^{3}}{\left(m_{1}+m_{2}\right)^{2}, P}$ & $m_{1}, m_{2}, a, R_{1}, T_{e 1}, L_{1}$ \\
& Interferometry & $\theta_{a}, i, \theta_{1}\left(\theta_{2}\right)$ & $\left(R_{2}, T_{e 2}, L_{2}\right)$ \\
& Photometry & Integrated flux $\int_{0}^{\infty} f_{\lambda} d \lambda$ & \\
\hline
\end{tabular}

of the potential of the combination of interferometric and spectroscopic data for DLSBs which is discussed further in Section 4.1.

\subsection{Cepheid Variables}

The distance to a Cepheid variable $d$ can be found by combining a measurement of the variation in angular diameter $\Delta \theta$, determined by interferometry, with the change in radius $\Delta R$, determined from spectroscopic radial velocity measurements, from

$$
d=\frac{2 \Delta R}{\Delta \theta}
$$

where $d$ is in the same units as $\Delta R$ and $\Delta \theta$ is in radians.

Equation 1 presents a simplistic relationship which, in reality, is significantly more complex. For example, in the case of the interferometric measurements, it is generally the angular diameter of the equivalent uniform disk that is determined and not the true limb-darkened angular diameter. Unfortunately the shape of the relationship between correlation and baseline for a limb-darkened disk and a uniform disk differ by only a few parts in a thousand, although the baseline scales differ, and it is generally impossible to determine the degree of limb-darkening from the interferometric data. For this reason measurements are generally fitted with the response for a uniformly illuminated disk and a correction, of the order of $10 \%$, based on theoretical model atmospheres is applied to obtain a value for the limb-darkened disk. There are difficulties associated with determining limb-darkening coefficients for Cepheids as a function of phase (Sasselov \& Karovska 1994) and, in the case of the complementary spectroscopy, there are difficulties associated with the measurement of the radial velocities and their conversion to pulsation velocities. These are discussed further in Section 4.2 . 
The measurement of $\Delta \theta$ for a Cepheid by interferometry has not yet been achieved although a mean angular diameter for $\delta$ Cep has been measured by Mourard et al. (1997). Nevertheless, several of the interferometers listed in Table 3 have the potential to measure the angular size variations of several Cepheids once they are fully commissioned.

\section{Interferometers and Interferometric Arrays}

Table 3 contains a list of optical/infrared interferometers and interferometric arrays. The instruments shown as having two apertures combine the light from only two apertures at a time. In most of these cases there is either an array of apertures, from which the two to be used can be selected, or there is the possibility of moving input optics between stations to provide a range of baselines. Two aperture instruments measure only the fringe visibility. The phase of the interference is not measured since it is completely corrupted by turbulence in the Earth's atmosphere. Images are not possible and symmetry in the brightness distribution across the source must be assumed. For angular diameter determinations and studies of binary systems this is generally of no concern - the relative brightness of the components of a binary system can be determined but with a $180^{\circ}$ ambiguity in the orientation of the system.

Instruments listed with three or more apertures are generally intended to combine the light from all the apertures simultaneously. In these array instruments the fringe visibility and phase are measured for each baseline and the phase-closure techniques, developed by radio astronomers, are used to circumvent the effects of atmospheric turbulence on the individual phases in order to produce images. This was first demonstrated with separated apertures, as distinct from aperture masks mounted on conventional telescopes, by Baldwin et al. (1996).

Two aperture interferometers and interferometric arrays both require dynamical optical path matching and techniques for overcoming the deleterious effects of the atmosphere such as the use of small apertures, wavefront tip-tilt correction and rapid signal sampling.

Astrometric interferometers and arrays are similar in principle but differ only in having extensive monitoring of the instrument stability by laser metrology and an optical path length compensator operating in vacuum. Astrometric interferometers are of two broad types - global and narrow angle (differential). Global astrometric interferometers, such as the NPOI Astrometric Array, measure the relative positions of stars over the whole sky with the aim of maintaining the Hipparcos frame and improving proper motion determinations by extending the timebase of measurements. Narrow angle interferometers are effectively double interferometers with each interferometer linked to the other by precision metrology. One interferometer is phase locked to a reference source while the second is locked to the target source to search for relative motion such as that produced by a faint unseen companion or giant planet.

Angel and Woolf (1997) have proposed an imaging nulling interferometer based on a concept proposed by Bracewell (1978) for the detection of exo-planets. By working in the infrared and suppressing the stellar flux, by combining the 
star's light out of phase, they argue that it should be possible to detect planets down to Earth size with an instrument operating in space.

The nulling interferometer and narrow-angle interferometers have been included in this outline of the types of interferometer because they offer the possibility of contributing the inclinations of planetary orbits and hence enabling planetary masses to be determined free of $\sin i$. There are technical challenges in developing these instruments and it is likely to be many years before scientific results are forthcoming. For this reason they, and space-based interferometry in general, are not discussed further here.

\section{Combining Interferometric and Spectroscopic Data}

In a discussion of the combination of interferometric and spectroscopic data for binary systems and Cepheids it must be recognised that every object will have different characteristics. Many will be more amenable to accurate measurements by one or other of the techniques but, ideally, the results of combining interferometric and spectroscopic data should not be dominated by the uncertainties due to either set of data.

The author is more familiar with the achievements and potential of interferometry and, in the following sections, the accuracy of interferometric measurements is discussed and the accuracy required in spectroscopic data for comparable contributions to the final uncertainties in the combined results for both binaries and Cepheids is outlined.

\subsection{Spectroscopic Binary Stars}

The accuracies now being achieved in ground based high angular resolution interferometry are impressive. In order to estimate the accuracies required from spectroscopy the following uncertainties in interferometrically determined parameters will be assumed: $\sigma_{i}< \pm 0.5^{\circ}, \sigma_{\theta_{a}}< \pm 1 \%$ and $\sigma_{\theta}< \pm 2 \%$. These are conservative figures and formal uncertainties down to $\pm 0.1^{\circ}$ in $i$ and less than $\pm 0.5 \%$ in $\theta_{a}$ have been obtained with the Mark III Interferometer (Hummel and Armstrong 1994). The uncertainties in stellar angular diameters $(\theta)$ determined by interferometry have been reviewed by Davis (1997) and uncertainties of $\pm 2 \%$ are now readily achieved with $\pm 1 \%$ achieved for a number of stars.

The masses of the components of a DLSB system are related to the spectroscopically determined parameters by

$$
\left(m_{1}+m_{2}\right) \propto\left(\frac{K_{1}+K_{2}}{\sin i}\right)^{3} P\left(1-\epsilon^{2}\right)^{\frac{3}{2}}
$$

where $K_{1}$ and $K_{2}$ are the half ranges of the radial velocities of the primary and secondary components respectively, $e$ is the eccentricity, and $P$ the orbital period. The ratio of the masses is given by $m_{1} / m_{2}=K_{2} / K_{1}$.

Ideally the uncertainty in $\left(K_{1}+K_{2}\right)$ should be comparable with that in $\sin i$. For the adopted uncertainty of $\pm 0.5^{\circ}$ in $i$, the uncertainty in $\sin i$ for $i>41^{\circ}$ is less than $\pm 1 \%$. The desired accuracy in $\left(K_{1}+K_{2}\right)$ for such systems is therefore also less than $\pm 1 \%$ but depends on the value of $i$. The accuracy in $\mathrm{m} \mathrm{s}^{-1}$ also depends on the magnitude of $\left(K_{1}+K_{2}\right)$ and it follows that the 
Table 3. Interferometers and Interferometric Arrays.

\begin{tabular}{|c|c|c|c|c|c|c|}
\hline No. & $\begin{array}{l}\text { Instrument } \\
\text { (Acronym) }\end{array}$ & Country & $\begin{array}{l}\text { Aperture } \\
\text { Diameter } \\
\text { (m) }\end{array}$ & $\begin{array}{l}\text { Longest } \\
\text { Baseline } \\
\text { (m) }\end{array}$ & $\begin{array}{l}\text { Wavelength } \\
\text { Range } \\
(\mu \mathrm{m})\end{array}$ & Status \\
\hline 1 & $\mathrm{SII}^{1}$ & Australia & $2 \times 6.8$ & 188 & 0.44 & Closed \\
\hline 2 & SUSI P'type ${ }^{2}$ & Australia & $2 \times 0.10$ & 11 & $0.4-0.5$ & Closed \\
\hline 3 & Mark $\mathrm{III}^{3}$ & USA & $2 \times 0.05$ & 32 & $0.45-0.8$ & Closed \\
\hline 4 & $\mathrm{I}^{2} \mathrm{~T}^{4}$ & France & $2 \times 0.26$ & 144 & Visible & Working \\
\hline 5 & $\mathrm{GI}_{2} \mathrm{~T}^{5}$ & France & $2 \times 1.5$ & 65 & Visible & Working \\
\hline 6 & ISI $^{6}$ & USA & $2 \times 1.65$ & 70 & 10 & Working \\
\hline 7 & SUSI $^{7}$ & Australia & $2 \times 0.14$ & 640 & $0.4-0.9$ & Working to $80 \mathrm{~m}$ \\
\hline 8 & IOTA $^{8}$ & USA & $3 \times 0.45$ & 38 & Visible/IR & Two apertures working \\
\hline 9 & COAST $^{\circ}$ & UK & $4 \times 0.4$ & 100 & Red/near IR & Working \\
\hline 10 & PTI $^{10}$ & USA & $2 \times 0.4$ & 100 & 2.2 & Working \\
\hline 11 & NPOI $(A A)^{11}$ & USA & $4 \times 0.125$ & 38 & $0.45-0.9$ & Working \\
\hline & NPOI $(I A)^{11}$ & & $6 \times 0.35$ & 437 & $0.45-0.9$ & Commissioning \\
\hline 12 & CHARA $^{12}$ & USA & $5 \times 1.0$ & 354 & $\begin{array}{l}0.55-0.9 \\
2.1-2.5\end{array}$ & Under construction \\
\hline 13 & ESO VLTI ${ }^{13}$ & Chile & $\begin{array}{l}4 \times 8 \\
3 \times 1.8\end{array}$ & 200 & $0.45-20$ & $\begin{array}{l}8 \mathrm{~m} \text { under construction } \\
\text { Design stage }\end{array}$ \\
\hline 14 & Keck $^{14}$ & USA & $\begin{array}{l}2 \times 10 \\
\text { Outriggers }\end{array}$ & 165 & $2.2-10$ & $\begin{array}{l}\text { Interferometry planned } \\
\text { Design stage }\end{array}$ \\
\hline
\end{tabular}

${ }^{1}$ Stellar Intensity Interferometer; Narrabri, Australia; Hanbury Brown, Davis \& Allen 1974, MNRAS, 167, 121

${ }^{2}$ SUSI Prototype (see 7); Sydney, Australia; Davis \& Tango 1985, Proc. Astron. Soc. Australia, 6,34

${ }^{3}$ Mark III Interferometer; Mt. Wilson, USA; Shao et al. 1988, A\&A, 193, 357

${ }^{4}$ Interféromètre à 2 Télescopes; Caussols, France; Koechlin 1988, ESO Proc., 29, 695

${ }^{5}$ Grand Interféromètre à 2 Télescopes; Mourard et al. 1994, A\&A, 283, 705

${ }^{6}$ Infrared Spatial Interferometer; Mount Wilson, USA; Bester, Danchi \& Townes 1990, Proc. SPIE, 1237, 40

${ }^{7}$ Sydney University Stellar Interferometer; Narrabri, Australia; Davis 1994, Proc. IAU Symposium 158,135

${ }^{8}$ Infrared-Optical Telscope Array; Mt. Hopkins, USA; Carleton et al. 1994, Proc. SPIE, 2200, 152

${ }^{9}$ Cambridge Optical Aperture Synthesis Telescope; Cambridge, UK; Baldwin et al. 1994, Proc. SPIE, 2200, 118

${ }^{10}$ Palomar Test Interferometer; Palomar, USA; Colavita et al. 1994, Proc. SPIE, 2200, 89

${ }^{11}$ Navy Prototype Optical Interferometer (AA = Astrometric Array: IA = Imaging Array); Flagstaff, USA; Armstrong et al. 1998, ApJ, 496, 550

${ }^{12}$ Center for High Angular Resolution Astronomy Array; Mt. Wilson, USA; McAlister et al. 1994, Proc. SPIE, 2200, 129

${ }^{13}$ European Southern Observatory Very Large Telescope Interferometer; Paranal, Chile; von der Lühe et al. 1994, Proc. SPIE. 2200, 168

${ }^{14}$ Keck Interferometer; Mauna Kea, USA; Colavita et al. 1994, Proc. SPIE, 2200, 89 
accuracy required in the spectroscopically determined radial velocities differs from one binary to the next and must be established on a case by case basis. Nevertheless, the accuracies required appear to be well within the capabilities of current spectroscopic techniques for the majority of systems. In determining the masses of the components of a binary system, the uncertainty in the eccentricity $(\epsilon)$, at least for low eccentricity orbits, is not a limiting factor.

Andersen (1991) has argued that masses need to be determined to significantly better than $\pm 5 \%$ to be useful. While the limiting sensitivities of the interferometers listed in Table 3 have not yet been established, it is anticipated that well in excess of one hundred double-lined systems will provide masses, with a significant number having accuracies in the 'useful' category. In selected cases masses with uncertainties $< \pm 2 \%$ should be possible.

In the case of SLSBs, spectroscopy yields the mass function and, in order to determine the individual masses, the sum of the masses must be found from Kepler's third law. This requires the orbital period and the semi-major axis $a$. The latter is found by combining the angular semi-major axis $\theta_{a}$ with the parallax $\pi$ of the system using

$$
a=\frac{\theta_{a}}{\pi}
$$

where $a$ is in $\mathrm{AU}$ for $\theta_{a}$ and $\pi$ in arcseconds.

Since the sum of the masses depends on the third power of the semi-major axis, the accuracy of the parallaxes is a key issue in establishing the accuracy of mass determinations for SLSBs. Hipparcos parallaxes for selected SLSBs, combined with interferometry and spectroscopy, should provide a significant number of accurate masses although this has not yet been examined in detail.

The distance to a DLSB system can be determined by combining interferometry and spectroscopy with an accuracy exceeding that of Hipparcos parallaxes for distances in excess of $\sim 10 \mathrm{pc}$. The distance $(d=1 / \pi)$ is obtained by combining the semi-major axis with the interferometrically determined angular semi-major axis using a rearranged equation 3 .

The semi-major axis is related to the spectroscopic parameters by

$$
a \propto\left(\frac{K_{1}+K_{2}}{\sin i}\right) P\left(1-\epsilon^{2}\right)^{\frac{1}{2}}
$$

Again, as in the case of mass determinations, the uncertainty in $\left(K_{1}+K_{2}\right)$ should ideally be comparable to that in $\sin i$. Given the potential accuracies in the contributing parameters in equations 3 and 4, uncertainties of less than $\pm 2 \%$ and, in favourable cases, $\pm 1 \%$ in $d$ should be possible. An uncertainty of $\pm 1 \%$ corresponds to an uncertainty of less than \pm 1 mas in the parallax for systems at distances exceeding $10 \mathrm{pc}$. As an example, the distance to $\alpha$ Vir was determined using observations obtained with the Stellar Intensity Interferometer (HerbisonEvans et al. 1971). Although the uncertainty in the distance was $\pm 5 \%$ ( $84 \pm$ $4 \mathrm{pc}$ ) this translates to an uncertainty in the parallax of only \pm 0.6 mas.

Distances determined as just described, or from Hipparcos, can be combined with the interferometrically determined angular diameters of component stars to give stellar radii. While the angular diameters of component stars in binary systems will not be determined with the same accuracy as for single stars, radii 
with uncertainties down to $\pm 2 \%$ should be possible in selected cases. Effective temperatures can be determined by combining angular diameters with integrated flux distributions, providing a reasonable determination of the flux from each component can be made, and this in turn will enable stellar luminosities to be calculated.

\subsection{Cepheid Variables}

The distance to a Cepheid can be found by the combination of interferometric and spectroscopic data using equation 1. Although uncertainties as small as $\pm 1 \%$ have been achieved in angular diameter measurements it is the variation in angular diameter $\Delta \theta$ that is required for Cepheid distance determinations. The accuracy achievable in $\Delta \theta$ will depend on the magnitude of the fractional change in radius $(\Delta R / R)$ of a Cepheid as it pulsates. It is estimated that the uncertainty in $\Delta \theta$ will be in the range $\pm 5 \%$ to $\pm 15 \%$ for some $60 \%$, and less than $\pm 20 \%$ for some $90 \%$ of Cepheids accessible to interferometry. It is anticipated that of the order of 50 Cepheids will become accessible in the next decade as the interferometers listed in Table 3 become fully operational.

The determination of $\Delta R$ from spectroscopy entails a number of difficulties that have been discussed by several authors (see for example Butler 1993, Sasselov \& Karovska 1994). Pulsation factors are used to convert the observed radial velocities, which are integrated over the entire observable stellar disk, to pulsation velocities. These are affected by the method used to determine the radial velocity from the observed line profile and by changing line asymmetries. These factors are not constant throughout the pulsation cycle (Butler 1993). The radial velocity determination itself depends on the lines used, the technique used for measuring the lines, the velocity gradient in the atmosphere, etc. Limb darkening affects the pulsation factor as well as the conversion of the observed angular diameter from a uniform disk value to the true limb-darkened angular diameter as discussed in Section 2.2.

The determination of distances to Cepheids by the combination of interferometric and spectroscopic data is clearly not a trivial task but it is, nevertheless, an important one with the potential to provide an independent calibration of the zero point of the Cepheid luminosity scale. The solution of the problems associated with the determination of the variation in linear radius from spectroscopy, and with the limb-darkening corrections to the interferometric angular diameters, requires the matching of observations with computer models of hydrodynamic non-LTE atmospheres (Sasselov \& Karovska 1994). This program has the potential to provide a reliable calibration of the zero point of the Cepheid distance scale to $\leq \pm 5 \%$ ( \pm 0.1 magnitudes).

\section{Summary}

The combination of interferometric and spectroscopic data for spectroscopic binary stars enables the simultaneous determination of radii, effective temperatures, masses and luminosities for individual stars. It also allows the determination of distances to the systems with accuracies surpassing Hipparcos parallaxes for distances greater than $10 \mathrm{pc}$. This is of particular significance for systems within clusters. In the case of Cepheids, the combination of data will lead to an 
independent calibration of the zero point of the Cepheid luminosity scale as well as the determination of radii, effective temperatures and luminosities throughout the pulsating cycles. Dynamic non-LTE modelling of the atmospheres is an important component of the Cepheid program.

It is undesirable that the uncertainty due to one technique should dominate the uncertainty in the results of the combination of data, and spectroscopists and interferometrists are encouraged to develop collaborative programs so that the potential of combining their data can be fulfilled.

Acknowledgments. The author is indebted to the IAU and the Science Foundation for Physics within the University of Sydney for travel grants that made attendance at IAU Colloquium 170 possible.

\section{References}

Andersen, J. 1991, ARA\&A, 3, 91

Angel, J.R.P., \& Woolf, N.J. 1997, ApJ, 475, 373

Armstrong, J.T., Mozurkewich, D., Vivekenand, M., Simon, R.S., Denison, C.S., Johnston, K.J., Pan, X.-P., Shao, M., \& Colavita, M.M. 1992, AJ, 104, 241

Baldwin, J.E. et al. (16 authors) 1996, A\&A, 306, L13

Bracewell, R.N. 1978, Nature, 274, 780

Butler, R.P. 1993, ApJ, 415, 323

Davis, J. 1979, in High Angular Resolution Stellar Interferometry (IAU Colloquium 50), J. Davis \& W.J. Tango, Sydney: University of Sydney Press, $1-1$

Davis, J. 1997, in Fundamental Stellar Properties: the Interaction between Observation and Theory (IAU Symposium 189), T.R. Bedding, A.J. Booth, \& J. Davis, Dordrecht: Kluwer, 31

ESA 1997, The Hipparcos and Tycho Catalogues, ESA SP-1200

Herbison-Evans, D., Hanbury Brown, R., Davis, J., \& Allen, L.R. 1971, MNRAS, 151,161

Hummel, C.A., \& Armstrong, J.T. 1994, in Very High Angular Resolution Imaging (IAU Symposium 158), J.G. Robertson \& W.J. Tango, Dordrecht: Kluwer, 410

Hummel, C.A., Armstrong, J.T., Quirrenbach, A., Buscher, D.F., Mozurkewich, D., Elias II, N.M., \& Wilson, R.E. 1994, AJ, 107, 1859

McAlister, H.A. 1979, in High Angular Resolution Stellar Interferometry (IAU Colloquium 50), J. Davis \& W.J. Tango, Sydney: University of Sydney Press, 3-1

McAlister, H.A. 1985, ARA\&A, 23, 59

Michelson, A.A. \& Pease, F.G. 1921, ApJ, 53, 249

Mourard, D., Bonneau, D., Koechlin, L., Labeyrie, A., Morand, F., Stee, P., Tallon-Bosc, I., \& Vakili, F. 1997, A\&A, 317, 789

Pan, X.P., Shao, M., Colavita, M.M., Mozurkewich, D., Simon, R.S., \& Johnston, K.J. 1990, ApJ, 356, 641 
Pan, X.P., Shao, M., Colavita, M.M., Armstrong, J.T., Mozurkewich, D., Vivekenand, M., Denison, C.S., Simon, R.S., \& Johnston, K.J. 1992, ApJ, 384, 624

Paresce, F., 1997, Science with the VLT Interferometer, Berlin: Springer-Verlag Sasselov, D. \& Karovska, M. 1994, ApJ, 432,367

\section{Discussion}

Walker: What do you estimate the $\Delta \mathrm{m}$ limit for binaries with SUSI to be?

Davis: We do not yet have an observational value, but estimate the limiting $\Delta m$ to be $\geq 3$ magnitudes. Christian Hummel gives $\Delta m$ as 3.5 to 4 magnitudes with the Mark III interferometer, based on experience with that instrument.

Tokovinin: It might not be unreasonable to try to measure Cepheid distances by looking at double Cepheids with periods of a few years. Of course, it would be difficult because the companions are faint. Measurements of not-too-close binaries with long baselines will lead to very high precision, and detection of perturbations due to close subsystems. There is some interesting science to be done in this way.

Davis: I agree. Laszlo Szabados will talk about Cepheid binaries later today. 